\title{
Asymptotically isochronous systems
}

\author{
Francesco Calogero $^{a, b}$ and David Gómez-Ullate ${ }^{c}$ \\ ${ }^{a}$ Dipartimento di Fisica, Università di Roma "La Sapienza", 00185 Rome, Italy. \\ E-mail: francesco.calogero@roma1.infn.it \\ ${ }^{b}$ Istituto Nazionale di Fisica Nucleare, Sezione di Roma. \\ ${ }^{c}$ Departamento de Física Teórica II, Universidad Complutense, 28040 Madrid, Spain. \\ E-mail: david.gomez-ullate@fis.ucm.es
}

Received November 19, 2008; Accepted December 20, 2008

\begin{abstract}
Mechanisms are elucidated underlying the existence of dynamical systems whose generic solutions approach asymptotically (at large time) isochronous evolutions: all their dependent variables tend asymptotically to functions periodic with the same fixed period. We focus on two such mechanisms, emphasizing their generality and illustrating each of them via a representative example. The first example belongs to a recently discovered class of integrable indeed solvable many-body problems. The second example consists of a broad class of (generally nonintegrable) models obtained by deforming appropriately the well-known (integrable and isochronous) many-body problem with inverse-cube two-body forces and a one-body linear ("harmonic oscillator") force.
\end{abstract}

\section{Introduction}

Isochronous phenomena, characterized by a time-recurrence with fixed periodicity, have intrigued observers from time immemorial. One of the first, semiquantitative observations of this phenomenology was reported by Galileo, who timed the motions of pendula in terms of the beating of his own pulse and thereby concluded that the oscillations of a pendulum seemed isochronous, namely periodic with a period independent from the initial conditions determining their amplitudes, provided they are small (in angular terms). This phenomenology - as well as its generality - was subsequently explained by Newton, whose contribution might well be considered to mark the beginning of modern physics - or at least of mathematical physics (the discipline providing the overall context of the present paper). Eventually the technology emerged of the isochronous systems par excellence clocks - playing a crucial role in the development of physics and other scientific disciplines, as well as in many important human activities (for instance navigation). Then, throughout the development of the modern theory of dynamical systems, much attention focussed on periodic, and more specifically on isochronous, evolutions. For instance in the context of the understanding of the interplay of regular and irregular time evolutions provided by KAM theory (see for instance [1]), the phenomenology associated with isochronous 
motions in the context of KAM theory has been rather recently investigated in [2]. An important field of activity where isochronous orbits arise is related to the study, connected with the 16th Hilbert problem, of the motions in the plane of a point circling around an isochronous center. A review of this field can be found in [3], and a few recent contributions in [4-6], some of them based on similar techniques to those referred to below.

A related line of research explores the connection between periodic orbits of hamiltonian dynamical systems and superintegrability (the existence of more integrals of motion than degrees of freedom). Nekhoroshev [7] proved that all confined orbits of a maximally superintegrable system are periodic. Some recent work on superintegrable systems and isochronous potentials (including many-body problems similar to those treated in this paper) can be found in [8-11].

A natural question that arises in classical isochronous systems concerns the properties of the spectrum of their quantum counterparts, in particular, whether the quantum systems whose classical counterparts are isochronous systems have equally spaced spectra. Some representative works that address this and other related problems can be found in [11-16]

Over the last three-four decades major progress occurred in the discovery and understanding of integrable dynamical systems with a finite or infinite number of degrees of freedom, and over the last decade the possibility was noticed and exploited to identify and investigate many isochronous dynamical systems characterized by a time evolution completely periodic (i. e., periodic in all degrees of freedom) with the same period. This isochronous evolution might prevail in the entire (natural) phase space of the model

under consideration (one talks then of an entirely isochronous system), implying of course that such a model is certainly integrable; or it might only prevail in an open (hence fully dimensional) region of its (natural) phase space, a phenomenology now known to characterize large families of nonintegrable dynamical systems possibly featuring quite complicated ("chaotic") behaviors outside the isochronous phase space region (for a review of these developments, see $[17,18]$ ). In the present paper we discuss another, perhaps more interesting, phenomenology, namely dynamical systems whose generic solutions approach asymptotically (at large time) isochronous evolutions: all their dependent variables tend asymptotically to functions periodic with the same fixed period. The definition of such dynamical systems is provided by the simultaneous validity of the two formulas

$$
\begin{aligned}
& \lim _{t \rightarrow+\infty}\left[z_{n}(t)-\tilde{z}_{n}(t)\right]=0, \quad n=1, \ldots, N, \\
& \tilde{z}_{n}(t+\tilde{T})=\tilde{z}_{n}(t), \quad n=1, \ldots, N .
\end{aligned}
$$

Notation: the $N$ (generally complex; but see below) numbers $z_{n}(t)$ denote the $N$ dependent variables of the dynamical system under consideration; we restrict consideration to the case when $N$ is a finite positive integer; the real variable $t$ denotes the time; the $N$ functions $\tilde{z}_{n}(t)$ characterize the asymptotic behavior of the dynamical system via (1a) and the periodicity requirement (1b) they satisfy characterizes the property of asymptotic isochronicity. This property is supposed to hold in an open (hence fully dimensional) region of the phase space of the dynamical system under consideration (possibly coinciding with its entire natural phase space): hence the dependent variables $z_{n}(t)$ denote here (the $N$ components of) a generic solution of the dynamical system evolving (at least for sufficiently large time) within that region, while the functions $\tilde{z}_{n}(t)$, which shall generally 
be different for different solutions $z_{n}(t)$, are required to satisfy the periodicity property (1b) with the fixed period $\tilde{T}$ (the same for all the solutions in the phase space region under consideration). Of course the formula (1a) does not define uniquely - for a given $N$-vector $\underline{z}(t)$ - a corresponding $N$-vector $\underline{\tilde{z}}(t)$ : the time-dependent $N$-vector $\underline{\tilde{z}}(t)$ is only identified by (1a) up to arbitrary corrections whose effects disappear in the asymptotic limit $t \rightarrow \infty$. The property of asymptotic isochronicity is guaranteed provided there exist just one $N$-vector $\underline{\tilde{z}}(t)$ satisfying both relations (1), for every generic solution $\underline{z}(t)$ in an open, fully dimensional, region of phase space - namely for every solution $\underline{z}(t)$ in that region of phase space, except possibly for some exceptional, generally singular, solutions belonging to a lower dimensional sector of that phase space region.

The elementary idea underlying the identification of large classes of such asymptotically isochronous dynamical systems is to start from isochronous systems and then modify them by introducing a deformation whose effects are significant through the time evolution yet disappear at large time: so that the modified systems loose their isochronous character (at finite times) but in some sense retain it (at large times) as the dominant feature characterizing their asymptotic behavior.

There are several possible ways to implement this strategy in order to manufacture asymptotically isochronous systems: some are rather trivial, some less so. This kind of judgement is of course subjective: for instance we tend to think that an important requirement for such systems to be deemed "interesting" is that they be autonomous because the interest of dynamical systems is also related to their potential usefulness in order to model natural phenomena, which are generally described by autonomous evolution equations - and moreover because the freedom to introduce instead an explicit time dependence in the equations of motion of a dynamical system would provide too easy a way to influence more or less at will the asymptotic behavior of such a system. But of course the difference between autonomous and nonautonomous systems is unessential, since any nonautonomous system can be made autonomous by treating time itself as an additional dependent variable.

In this paper we focus on two mechanisms yielding asymptotically isochronous systems, and illustrate each of them via a representative example. The first example (see Section 2) belongs to a recently discovered class of integrable indeed solvable many-body problems [19]; in this case we eventually focus on as simple and specific an example as possible, which is also suitable to exhibit some numerical results - but we trust our presentation is adequate to illustrate the generality of the approach. In this case the periodic behavior prevailing asymptotically corresponds to a special solution of the dynamical system under consideration belonging to a region of phase space with positive codimension - albeit not an isolated solution of this system, so not quite identifiable as a limit cycle. Hence this model might be considered a representative example of a phenomenology characterized by the presence of some kind of friction. The second example (see Section 3) consists of a broad class of models obtained by deforming appropriately the well-known (see for instance [20]) integrable and isochronous one-dimensional many-body problem with inverse-cube two-body forces and a one-body linear ("harmonic oscillator") force; the alert reader will again appreciate the generality of the approach, even though we illustrate it by focusing on a specific model (also restricting consideration to real dependent variables). In this second case the time-dependent $N$-vector to which the solutions of the model tend asymptotically is not restricted to be in a sector of phase space with positive 
codimension and is generally not itself a solution of the asymptotically isochronous $\mathrm{N}$ body model, so this phenomenology does not correspond to what is generally referred to as a limit cycle behavior. In each of these two cases we back the qualitative understanding of the origin of the relevant phenomenology with a proof of its actual emergence, see (1). A section entitled "Outlook" in which we elaborate tersely on the generality of this phenomenology concludes the paper.

\section{An asymptotically isochronous class of solvable many- body problems}

A particular mechanism to manufacture integrable, indeed solvable, dynamical systems interpretable as many-body problems inasmuch as they are characterized by Newtonian equations of motion ("acceleration equal force") was introduced about three decades ago [21] and has been subsequently exploited to identify and investigate several such systems (for reviews of these developments see for instance $[18,20]$ ). The idea is to exploit the nonlinear relation among the $N$ coefficients $c_{m}(t)$ of a (for definiteness, monic) timedependent polynomial of degree $N$ and its $N$ zeros $z_{n}(t)$ :

$$
\psi(z, t)=z^{N}+\sum_{m=1}^{N} c_{m}(t) z^{N-m}=\prod_{n=1}^{N}\left[z-z_{n}(t)\right] .
$$

A class of such systems is characterized by the fact that the $N$ coefficients $c_{m}(t)$ evolve in time according to a system of linear second-order constant-coefficient ODEs, the solution of which is a purely algebraic task (requiring essentially the diagonalization of an explicitly known matrix of order $N$ ). The determination of the corresponding time evolution of the $N$ zeros $z_{n}(t)$ is therefore as well a purely algebraic task: computing the $N$ zeros of a known polynomial. And it so happens that in many cases $[18,20,21]$ this time evolution is indeed interpretable as that characterizing a Newtonian $N$-body problem - hence a solvable $N$-body problem, since its solution can be achieved by purely algebraic means.

Indeed the solution $z_{n}(t)$ of such a model is reduced to finding the $N$ zeros of a polynomial of degree $N$ in the (complex) variable $z$, see (2), whose coefficients $c_{m}(t)$ generally evolve exponentially in time, typically

$$
c_{m}(t)=\sum_{\ell=1}^{N}\left\{\gamma^{(\ell,+)} u_{m}^{(\ell,+)} \exp \left[\lambda^{(\ell,+)} t\right]+\gamma^{(\ell,-)} u_{m}^{(\ell,-)} \exp \left[\lambda^{(\ell,-)} t\right]\right\},
$$

where the $2 N$ constants $\gamma^{(\ell, \pm)}$ are arbitrary (determined by the initial data $z_{n}(0), \dot{z}_{n}(0)$ in the context of the initial-value problem for the $N$-body system) and the $2 N$ numbers $\lambda^{(\ell, \pm)}$ respectively the quantities $u_{m}^{(\ell, \pm)}$ are the eigenvalues respectively the (components of the) eigenvectors of the matrix eigenvalue problem characterizing, as explained above, the dynamics of this system. Note that these eigenvalues and eigenvectors are associated to the dynamical problem under consideration: they do not depend on the initial data identifying a particular solution, namely they are the same for all the solutions of the system. 
It is now clear (and indeed well known $[18,20,21]$ ) that if the $2 N$ eigenvalues $\lambda^{(\ell, \pm)}$ are all integer multiples of a single imaginary number $i \omega$ (with $\omega>0$ ), $\lambda^{(\ell, \pm)}=i k^{(\ell, \pm)} \omega$ with the $2 N$ numbers $k_{\ell}^{( \pm)}$arbitrary integers (positive or negative, but not vanishing), then the polynomial $\psi(z, t)$ is clearly periodic with the (possibly nonprimitive) period

$$
\begin{aligned}
& T=\frac{2 \pi}{\omega}, \\
& \psi(z, t+T)=\psi(z, t),
\end{aligned}
$$

hence all its zeros $z_{n}(t)$ are as well periodic with this same period or possibly with a (generally small [22]) integer multiple $p$ of this period, $\tilde{T}=p T$, due to the possibility that they exchange their role through the time evolution. Hence the corresponding $N$-body problem is isochronous.

And it is as well plain that if, out of the $2 N$ eigenvalues $\lambda^{(\ell, \pm)}$, only a (nonempty) subset have the property indicated above while all the others feature a negative real part, then the many-body problem in question is asymptotically isochronous. This observation is not new, see for instance Section 4.2.3 of Ref. [20] (entitled "Some special cases: models with a limit cycle, models with confined and periodic motions, Hamiltonian models, translation-invariant models, models featuring equilibrium and spiraling configurations, models featuring only completely periodic motions"); but, to the best of our knowledge, this mechanism yielding asymptotically isochronous many-body problems was never analyzed in explicit detail (including the display of numerical results). This is what we do in this section, by focusing on a specific model whose integrable, indeed solvable, character has been ascertained only quite recently [19].

\subsection{A specific example}

This $N$-body problem (with $N \geq 3$ ) is characterized by the Newtonian equations of motion

$$
\begin{array}{r}
\ddot{z}_{n}=-a_{1} \dot{z}_{n}+a_{2} z_{n} \frac{z_{n}^{2}-5}{z_{n}^{2}-1}-2 a_{3} \frac{z_{n}^{2}+1}{z_{n}^{2}-1}-2 a_{4} z_{n} \\
+2 \sum_{m=1, m \neq n}^{N} \frac{\dot{z}_{n} \dot{z}_{m}+a_{2}+a_{3} z_{n}+a_{4}\left(z_{n}^{2}-1\right)}{z_{n}-z_{m}}, n=1, \ldots, N,
\end{array}
$$

where the 4 "coupling constants" $a_{j}$ are a priori arbitrary complex numbers, superimposed dots denote time-differentiations and the rest of the notation is self-evident. The solvable character of this $N$-body problem hinges [19] upon the following 4 restrictions on its initial data:

$$
\sum_{n=1}^{N} \frac{1}{z_{n}(0) \pm 1}=0, \quad \sum_{n=1}^{N} \frac{\dot{z}_{n}(0)}{\left[z_{n}(0) \pm 1\right]^{2}}=0,
$$

which are then sufficient [19] to guarantee that, throughout the time evolution,

$$
\sum_{n=1}^{N} \frac{1}{z_{n}(t) \pm 1}=0
$$


implying that for this model it is justified to assume that only the evolution of $N-2$ particles is determined by the Newtonian equations of motion (5a), while the evolution of the remaining two is determined by these conditions, see $(5 \mathrm{c})$.

Then the evolution of the $N$ "particle coordinates" $z_{n}(t)$ - taking generally place in the complex $z$-plane - coincides with the evolution of the $N$ zeros of a monic polynomial of degree $N$ in the variable $z$ analogous to $\psi(z, t)$, see $(2)$, but more specifically reading as follows [19]:

$$
\begin{aligned}
& \psi(z, t)=\pi_{N}(z)+\sum_{m=1}^{N-3}\left[c_{m}(t) \pi_{N-m}(z)\right]+c_{N}(t), \\
& \pi_{m}(z)=z^{m}-\varepsilon_{m} \frac{m}{2} z^{2}-\varepsilon_{m+1} m z, \quad m=0,1, \ldots, N, \\
& \varepsilon_{m}=1 \text { if } m \text { is even }, \quad \varepsilon_{m}=0 \text { if } m \text { is odd. }
\end{aligned}
$$

And the coefficients $c_{m}(t)$ evolve indeed according to formulas analogous to (3), but more specifically reading as follows [19]:

$$
\begin{aligned}
c_{m}(t) & =\sum_{\ell=1, \ell \neq N-1, N-2}^{N}\left\{\gamma^{(\ell,+)} u_{m}^{(\ell,+)} \exp \left[\lambda^{(\ell,+)} t\right]+\gamma^{(\ell,-)} u_{m}^{(\ell,-)} \exp \left[\lambda^{(\ell,-)} t\right]\right\} \\
m & =1, \ldots, N-3 \text { and } m=N \\
\lambda^{(\ell, \pm)} & =\frac{-a_{1} \pm \Delta_{\ell}}{2}, \quad \Delta_{\ell}^{2}=a_{1}^{2}+4 \ell\left[a_{2}+(2 N-\ell-3) a_{4}\right] \\
\ell & =1, \ldots, N-3, N .
\end{aligned}
$$

Note that the coupling constant $a_{3}$ does not appear explicitly in these formulas, but of course all 4 coupling constants $a_{j}$ do play a role in determining the quantities $u_{m}^{(\ell, \pm)}$ appearing in the right-hand side of (7a).

We now restrict attention to the $N=3$ case, since this is sufficient, indeed convenient, for exhibiting quite explicitly an asymptotically isochronous model. Then the only relevant coefficient (see (7a)) is

$$
\begin{aligned}
& c_{3}(t)=\gamma_{+} \exp \left(\lambda_{+} t\right)+\gamma_{-} \exp \left(\lambda_{-} t\right), \\
& \lambda_{ \pm}=\frac{-a_{1} \pm \Delta}{2}, \quad \Delta^{2}=a_{1}^{2}+12 a_{2},
\end{aligned}
$$

where the somewhat simplified notation we are now using is we trust self-explanatory (and note that in this case with $N=3$ the eigenvalues $\lambda_{ \pm}$only depend on the two coupling constants $a_{1}$ and $a_{2}$ ). Correspondingly, the positions of the 3 moving particles are the 3 zeros $z_{n}(t)$ of the third-degree polynomial

$$
\psi(z, t)=\pi_{3}(z)+c_{3}(t)=z^{3}-3 z+c_{3}(t)=\prod_{n=1}^{3}\left[z-z_{n}(t)\right] .
$$

Note that these 3 zeros automatically satisfy the requirements (5c), which corresponds [19] to the condition that the partial derivative of $\psi(z, t)$ with respect to $z$ vanish at $z= \pm 1$, $\psi_{z}( \pm 1, t)=0$. 
Assume now that the two coupling constants $a_{1}$ and $a_{2}$ entail, via (8b),

$$
\lambda_{+}=i \omega, \quad \lambda_{-}=-\alpha+i \beta,
$$

with $\alpha$ positive, $\alpha>0, \omega$ also positive, $\omega>0$ (for definiteness), and $\beta$ real but otherwise arbitrary. This indeed happens provided

$$
a_{1}=\alpha-i(\beta+\omega), \quad a_{2}=\frac{\omega(\beta+i \alpha)}{3} .
$$

It is now plain that the asymptotic condition (1a) holds now with $\tilde{z}_{n}(t)$ being the three roots of the polynomial $z^{3}-3 z+\gamma_{+} \exp (i \omega t)$,

$$
z^{3}-3 z+\gamma_{+} \exp (i \omega t)=\prod_{n=1}^{3}\left[z-\tilde{z}_{n}(t)\right],
$$

which provide of course also the special solution of the model (5) (with $N=3$ ) corresponding to initial data such that $\gamma_{-}$vanishes (see (8a)). And it is as well plain that the time evolution of this polynomial is periodic with period $T$, see (4a), hence the corresponding evolution of each of its 3 zeros is clearly periodic with periods $T, 2 T$ or $3 T$, depending whether that zero does not "exchange its role" through the motion with another zero or does so with one or with both the other two zeros.

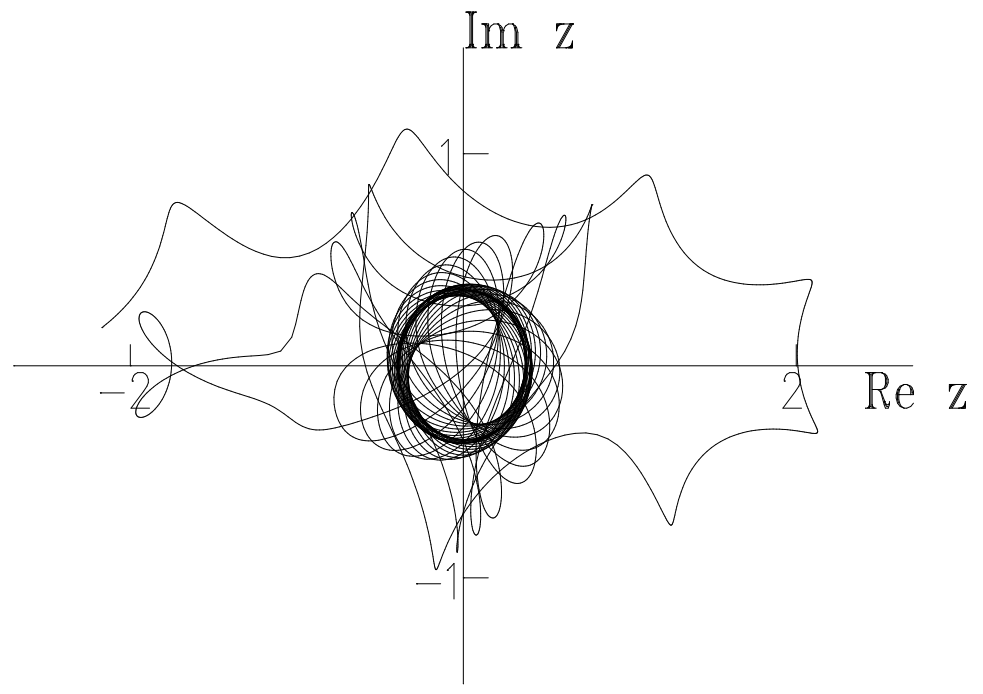

Figure 1. Trajectory of $z_{1}(t)$ in the complex $z$-plane from $t=0$ to $t=50$ (see text)

We complete this section by displaying one specific example, namely the solution of the system of ODEs (5) with $N=3, \omega=2 \pi$ implying $T=1$ (see (4a)), $a_{3}=a_{4}=0, a_{1}$ and $a_{2}$ given by (10) with $\alpha=0.1$ and $\beta=-3$, and with initial data

$$
\begin{array}{ll}
z_{1}(0)=-2.1702823+0.18021431 \mathrm{i}, & \dot{z}_{1}(0)=1.2487698+0.76941297 \mathrm{i}, \\
z_{2}(0)=0.71910399-0.89149288 \mathrm{i}, & \dot{z}_{2}(0)=-2.7507203+1.3102500 \mathrm{i}, \\
z_{3}(0)=1.4511783+0.71127857 \mathrm{i}, & \dot{z}_{3}(0)=1.5019505-2.0796630 \mathrm{i},
\end{array}
$$




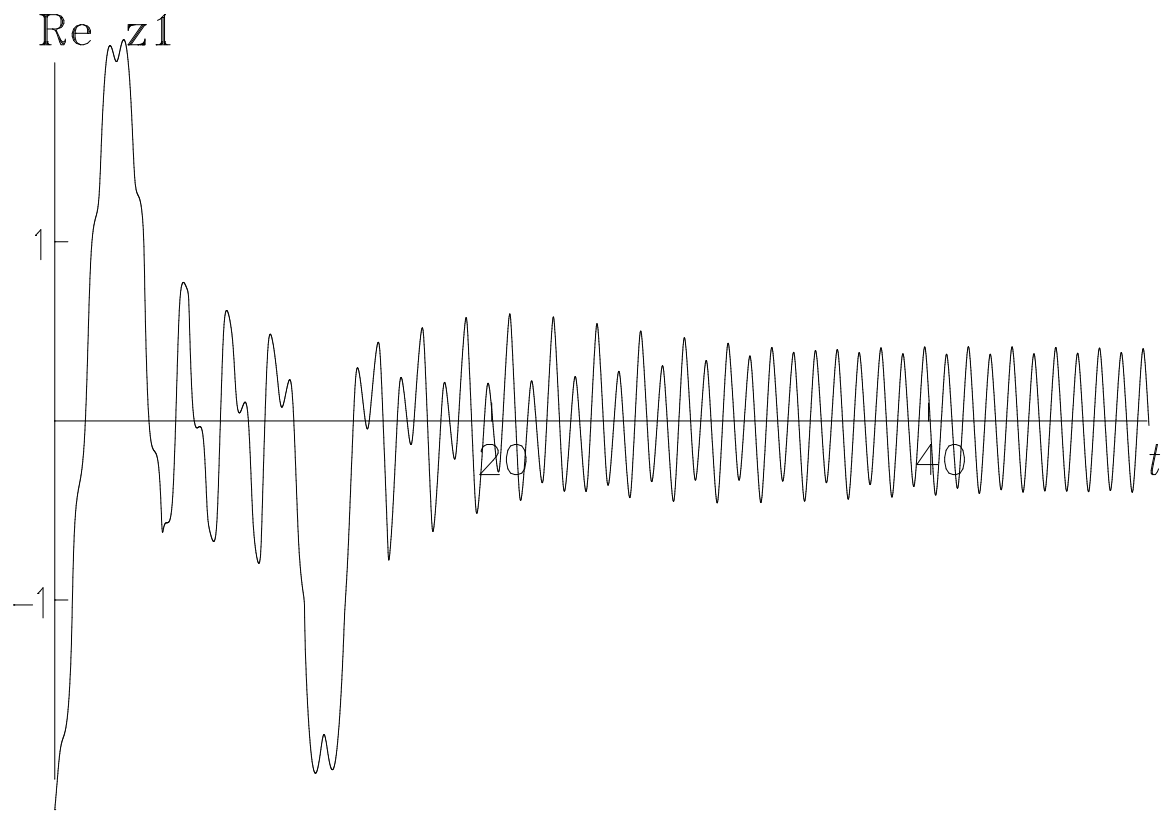

Figure 2. Plot of Re $z_{1}$ as a function of $t$ (see text)

satisfying the conditions (5b) and entailing $\gamma_{+}=0.5+i, \gamma_{-}=3-3 i$ (see (8a)). The results displayed are, from $t=0$ to $t=50$, the trajectory of $z_{1}(t)$ in the complex $z$-plane (Fig. 1), the real part of $z_{1}(t)$ as a function of $t$ (Fig. 2) (the behavior of the imaginary part is qualitatively analogous) and the evolution of the quantity

$$
D(t)=\left|c_{3}(t)-\gamma_{+} \exp (i \omega t)\right| \quad \text { with } \quad c_{3}(t)=-z_{1}(t) z_{2}(t) z_{3}(t)
$$

(Fig. 3) that clearly provides a measure of the distance of this solution $\underline{z}(t)$ from its periodic limit $\underline{\tilde{z}}(t)$ (see (8c) and (11), as well as (1)). The numerical integration has been performed with an embedded Runge-Kutta method of order $8(5,3)$ with automatic step size control, as developed by Prince and Dormand [23]; the integration and the graphical output have been performed with the software DyNAmics Solver developed by J. Aguirregabiria. ${ }^{1}$ The results displayed have been obtained by integrating numerically the system of ODEs (5), checking throughout the integration the validity of the conditions $(5 c)$ as well as the two conditions

$$
z_{1}(t)+z_{2}(t)+z_{3}(t)=0, \quad z_{1}(t) z_{2}(t)+z_{2}(t) z_{3}(t)+z_{3}(t) z_{1}(t)=-3
$$

(see $(8 \mathrm{c})$ ). The results reported are just a representative example of several numerical computations we did with different parameters and initial data, computations which were found to be quite reliable and stable unless the time evolution entailed a near collision of particles or their passage close to the special values $z= \pm 1$ (see (5a)).

\footnotetext{
${ }^{1}$ This software is available at http://tp.lc.ehu.es/jma/ds/ds.html
} 


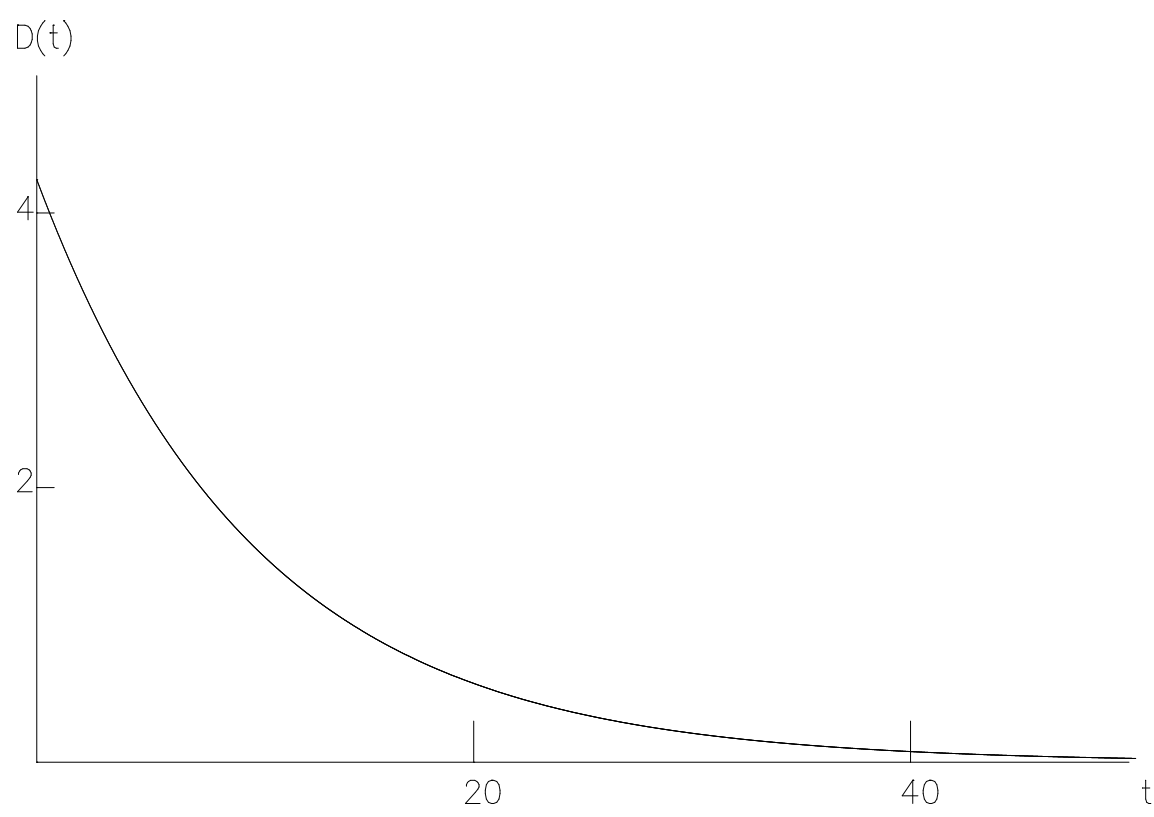

Figure 3. Plot of the distance $D(t)$ (see (12))

\section{A (generally nonintegrable) class of asymptotically isochronous many-body models}

In this section we consider a class of asymptotically isochronous models obtained by deforming the well-known integrable $N$-body problem with two-body inverse cube forces and a one-body linear force, which is of course isochronous when no deformation is present [20]. In particular we focus on the following equations of motion:

$$
\begin{aligned}
& \ddot{x}_{n}+\frac{1}{4} \omega^{2} x_{n}=g^{2} \sum_{m=1, m \neq n}^{N}\left(x_{n}-x_{m}\right)^{-3}+F(w, \underline{x}, \underline{\dot{x}}), \quad n=1, \ldots, N, \\
& \dot{w}=w[\alpha \log w-f(w, \underline{x}, \underline{\dot{x}})],
\end{aligned}
$$

with

$$
0<w(0)<1 .
$$

Here $N$ is an arbitrary positive integer $(N \geq 2)$; the $N$ dependent variables $x_{n} \equiv x_{n}(t)$ may be interpreted as the coordinates of $N$ particles evolving according to the Newtonian ("acceleration equal force") equations of motion (14a); these variables $x_{n}$ are hereafter assumed to be all real (until we mention below to what extend the results change if the variables $x_{n}$ are allowed to be complex), and $\underline{x}$ denotes of course the $N$-vector with components $x_{n}$ (this has motivated the notational replacement of the particle coordinates $z_{n}$ with $x_{n}$, to be kept in mind when comparing the formulas written in this section with those written in the preceeding sections); likewise the auxiliary dependent variable 
$w \equiv w(t)$ evolves according to the first-order ODE (14b) with an initial condition satisfying the inequalities (14c) (but clearly, see below, one could replace this first-order ODE with an appropriate second-order "Newtonian" ODE); $t$ denotes of course the (real) independent variable ("time": ranging from the initial time $t=0$ to the asymptotic time $t=+\infty$ ), and superimposed dots denote again differentiations with respect to this variable; $\omega, g^{2}$ and $\alpha$ are three positive (but otherwise arbitrary) constants; the main restriction on the, otherwise arbitrary, function $F(w, \underline{x}, \underline{v})$ is that it vanish when $w$ vanishes,

$$
F(0, \underline{x}, \underline{v})=0,
$$

and the main restrictions on the function $f(w, \underline{x}, \underline{v})$ is that it entail via $(14 \mathrm{~b})$ a (very fast: see below) asymptotic vanishing (as $t \rightarrow+\infty$ ) of the auxiliary variable $w(t)$,

$$
\lim _{t \rightarrow+\infty}[w(t)]=0 .
$$

A condition generally sufficient (but by no means necessary) to cause this is clearly (see $(14 \mathrm{~b})$ with (14c) and below) the requirement that $f(w, \underline{x}, \underline{v})$ be finite and nonnegative,

$$
0 \leq f(w, \underline{x}, \underline{v}) \leq a^{2},
$$

for all (real) values of $w, \underline{x}$ and $\underline{v}$; it is indeed plain (for a proof, see below) that these conditions together with (14b) entail the inequalities

$$
0<w(t) \leq[w(0)]^{\exp (\alpha t)},
$$

hence (see (14c) and recall that $\alpha>0$ ) the auxiliary variable $w(t)$ is always positive and vanishes asymptotically faster than exponentially,

$$
\lim _{t \rightarrow+\infty}[w(t) \exp (b t)]=0
$$

with $b$ any arbitrary constant. Restrictions on the dependence of the function $F(w, \underline{x}, \underline{v})$ upon the $N$-vectors $\underline{x}$ and $\underline{v}$ are also required: a simple sufficient (but of course not necessary) condition, also encompassing (15), is that there exist a finite (positive) constant $C$ and a positive number $\beta$ such that

$$
|F(w, \underline{x}, \underline{v})| \leq C|w|^{\beta}, \quad \beta>0,
$$

for all (real) values of $w, \underline{x}$ and $\underline{v}$. Functions satisfying these conditions are for instance

$$
\begin{aligned}
& F(w, \underline{x}, \underline{v})=C w^{\beta}\left[1+\sum_{n=1}^{N}\left(A_{n}^{2} x_{n}^{2}+B_{n}^{2} v_{n}^{2}\right)\right]^{-1} \\
& F(w, \underline{x}, \underline{v})=C w^{\beta} \exp \left[-\sum_{n=1}^{N}\left(A_{n}^{2} x_{n}^{2}+B_{n}^{2} v_{n}^{2}\right)\right]
\end{aligned}
$$

where $A_{n}$ and $B_{n}$ are arbitrary real constants.

Our main result states that, for every ( $N$-vector) solution $\underline{x}(t)$ of this dynamical system, an $(N$-vector) $\underline{\tilde{x}}(t)$ characterizing its asymptotic behavior (as $t \rightarrow+\infty)$ via the formula (1a) (exists and) has the property to be completely periodic (i. e., periodic with the same 
period in all its components), see (1b) with $\tilde{T}=T$, see (4a). Of course this asymptotic $N$-vector $\underline{\tilde{x}}(t)$ will depend on the solution $\underline{x}(t)$ under consideration - in particular, it will depend on the initial data, $\underline{x}(0)$ and $\underline{\dot{x}}(0)$, determining that solution in the context of the initial-value problem for the $N$-body problem (14): but let us re-emphasize that, for any arbitrary choice of these data (of course, satisfying the condition $x_{n}(0) \neq x_{m}$ (0) for $n \neq m$, see (14a)) it shall feature the property (1), namely all solutions $\underline{x}(t)$ of the system (14) shall feature the property of completely isochronous asymptotic periodicity (1) (with $\tilde{T}=T$, see $(4 \mathrm{a}))$.

This result is a natural consequence of the well-known fact (see for instance [20]) that all solutions of the system of Newtonian equations (14a) without the $F$ term in the right-hand side are completely periodic with period $T$, see (4a), namely they all feature themselves the property (1b) with $\tilde{T}=T$. It stands therefore to reason that, if the function $F(w, \underline{x}, \underline{v})$ vanishes when $w$ vanishes, see (15), and if the time evolution (14b) of the auxiliary variable $w(t)$ entails that this dependent variable indeed vanishes asymptotically, see (16a), fast enough (see (16d)), then asymptotically all solutions of our model (14) shall behave as the solutions of the same model without the $F$ term, entailing the asymptotic phenomenology (1) with $\tilde{T}=T$, see (4a).

To turn this hunch into a theorem a proof must be provided. This we do in the following subsection. Then in Section 4 we tersely discuss, again in the same qualitative vein as done above, to what extent the phenomenology described in this paper, and shown to occur in a specific, representative model, can be expected to occur in more general contexts.

\subsection{A theorem and its proof}

Theorem 1. The conditions (16b) and (17) are sufficient to guarantee that every solution of the $N$-body problem (14) with the three constants $\omega, g^{2}$ and $\alpha$ all positive yield the outcomes (16a) and (1) with $\tilde{T}=T$, see (4a); in particular they guarantee that there exists, corresponding to every solution $\underline{x}(t)$ of the $N$-body problem (14), an $N$-vector $\underline{\tilde{x}}(t)$ satisfying both formulas (1) (of course, with $z_{n}$ replaced by $x_{n}$ and $\tilde{z}_{n}$ by $\tilde{x}_{n}$ ).

Proof. First of all let us prove the inequalities (16c), obvious as they are. To this end we set

$$
w(t)=[w(0)]^{\exp [\varphi(t)]},
$$

so that

$$
\varphi(0)=0
$$

and (from (14b))

$$
\dot{\varphi}(t)=\alpha+f[w(t), \underline{x}(t), \underline{\dot{x}}(t)] \exp [-\varphi(t)]|\log [w(0)]|^{-1},
$$

where we used the fact that $\log [w(0)]=-|\log [w(0)]|$, see (14c). This ODE, together with the initial datum (18b) and the inequalities (16b), clearly imply that $\varphi(t)$ is positive and finite for $0 \leq t<\infty$, indeed validity of the inequalities

$$
\alpha t<\varphi(t)<\infty, \quad 0 \leq t<\infty,
$$


which, via (18a) and (14c), yield (16c).

Next, let us introduce the counterpart of the Newtonian equations of motion (14a), but without the $F$ term in the right-hand side:

$$
\ddot{\tilde{x}}_{n}+\frac{1}{4} \omega^{2} \tilde{x}_{n}=g^{2} \sum_{m=1, m \neq n}^{N}\left(\tilde{x}_{n}-\tilde{x}_{m}\right)^{-3}, \quad n=1, \ldots, N \text {. }
$$

Here it is justified to use the notation $\tilde{x}_{n} \equiv \tilde{x}_{n}(t)$ for the dependent variables, since it is well-known [20] that all the solutions of this Newtonian $N$-body problem are completely periodic with period $T$, see (4a), consistently with (1b) with $\tilde{T}=T$.

Let us now remark that, due to the strict positivity of $g^{2}$, this system of ODEs entails that

$$
\left|\tilde{x}_{n}(t)-\tilde{x}_{m}(t)\right|>\tilde{c}^{2}, \quad \tilde{c}^{2}>0, \quad n \neq m, \quad 0 \leq t<\infty,
$$

where $\tilde{c}^{2}$ is a time-independent constant that generally depends on the particular solution under consideration but is certainly strictly positive, $\tilde{c}^{2}>0$. Likewise, again due to the strict positivity of $g^{2}$, the system of ODEs (14a) with (17) and (16c) (entailing $\left.|F(w, \underline{x}, \underline{v})| \leq D, D=C|w(0)|^{\beta}\right)$ implies that

$$
\left|x_{n}(t)-x_{m}(t)\right|>c^{2}, \quad c^{2}>0, \quad n \neq m, \quad 0 \leq t<\infty,
$$

where $c^{2}$ is again a time-independent constant that generally depends on the particular solution under consideration but is certainly strictly positive, $c^{2}>0$. Moreover the systems of ODEs (19) and (14) with (17) and (16c) clearly imply that, for all (finite, positive) time, the functions $\tilde{x}_{n}(t)$ and $x_{n}(t)$ are finite.

Let us now set

$$
\xi_{n}(t)=x_{n}(t)-\tilde{x}_{n}(t)
$$

These functions $\xi_{n}(t)$ satisfy - as implied by subtracting (19) from (14a) - the system of ODEs

$$
\ddot{\xi}_{n}+\frac{1}{4} \omega^{2} \xi_{n}+g^{2} \sum_{m=1, m \neq n}^{N}\left[\xi_{n}-\xi_{m}\right] \varphi_{n m}(\underline{x}, \underline{\tilde{x}})=F[w, \underline{x}, \underline{\dot{x}}]
$$

with

$$
\varphi_{n m}(\underline{x}, \underline{\tilde{x}})=\frac{\left(x_{n}-x_{m}\right)^{2}+\left(x_{n}-x_{m}\right)\left(\tilde{x}_{n}-\tilde{x}_{m}\right)+\left(\tilde{x}_{n}-\tilde{x}_{m}\right)^{2}}{\left(x_{n}-x_{m}\right)^{3}\left(\tilde{x}_{n}-\tilde{x}_{m}\right)^{3}} .
$$

Note that the above bounds, (20), as well as the finiteness of $x_{n}$ and $\tilde{x}_{n}$ for all (positive) time, guarantee that these functions $\varphi_{n m}(\underline{x}, \underline{\tilde{x}})$ remain finite for all time, namely that there always exist time-independent finite upper and lower bounds $\varphi_{ \pm}$satisfied by them for all time,

$$
\varphi_{-} \leq \varphi_{n m}(\underline{x}, \underline{\tilde{x}}) \leq \varphi_{+} .
$$


These bounds depend of course on the particular solutions $\underline{x}$ and $\underline{x}$ under consideration, but let us re-emphasize that, for any such solutions, they are finite.

It is now clear that the theorem is proven if we can show that this system of ODEs admits a solution satisfying the asymptotic condition

$$
\lim _{t \rightarrow+\infty}\left[\xi_{n}(t)\right]=0, \quad n=1, \ldots, N
$$

(see (1a) and (21)). As can be easily verified such a solution of (22) is provided by the formula

$$
\xi_{n}(t)=\int_{t}^{\infty} d t^{\prime} F\left[w\left(t^{\prime}\right), \underline{x}\left(t^{\prime}\right), \underline{\dot{x}}\left(t^{\prime}\right)\right] G_{n}\left(t, t^{\prime}\right), \quad n=1, \ldots, N,
$$

where the functions $G_{n}\left(t, t^{\prime}\right)$ are the Green's functions associated with the left-hand side of the system of ODEs (22a), namely the solutions of the system of ODEs

$$
\begin{array}{r}
\frac{\partial^{2} G_{n}\left(t, t^{\prime}\right)}{\partial t^{2}}+\frac{1}{4} \omega^{2} G_{n}\left(t, t^{\prime}\right) \\
+g^{2} \sum_{m=1, m \neq n}^{N}\left[G_{n}\left(t, t^{\prime}\right)-G_{m}\left(t, t^{\prime}\right)\right] \varphi_{n m}[\underline{x}(t), \underline{\tilde{x}}(t)]=0, \quad t \leq t^{\prime}, \\
G_{n}(t, t)=0,\left.\quad \frac{\partial G_{n}\left(t, t^{\prime}\right)}{\partial t}\right|_{t=t^{\prime}}=-1, \quad n=1, \ldots, N .
\end{array}
$$

Indeed, while these Green functions cannot be computed explicitly (since we do not know the $N$-vectors $\underline{x}(t)$ and $\underline{\tilde{x}}(t)$, hence neither the functions $\left.\varphi_{n m}[\underline{x}(t), \underline{\tilde{x}}(t)]\right)$, it is plain from the linear character of this system of ODEs and from the bounds $(22 \mathrm{c})$ that these Green functions can grow (in modulus) at most exponentially as $t \rightarrow \infty$ and/or $t^{\prime} \rightarrow \infty$; so that the faster than exponential vanishing of $F\left[w\left(t^{\prime}\right), \underline{x}\left(t^{\prime}\right), \underline{\dot{x}}\left(t^{\prime}\right)\right]$ as $t^{\prime} \rightarrow \infty$ (implied by (17) with (16d)) entails that the integral in the right-hand side of the solution formula (24a) vanishes asymptotically (as $t \rightarrow \infty$ ).

Remark. It is clear how this example could have been made more general by allowing the function $F$ appearing in the right hand side of (14a) to depend on the index $n$, and/or by replacing the single auxiliary variable $w(t)$ by a $J$-vector $\underline{w}(t)$ with $J$ an arbitrary positive integer, and so on; without invalidating our conclusion, but complicating our proof. Let us also re-emphasize that the hypotheses made above to prove this theorem are sufficient but by no means necessary for its validity. More specific, and possibly considerably less stringent, conditions yielding an analogous conclusion can and will be introduced whenever this kind of result shall be considered in specific (possibly applicative) contexts. Our motivation to assume here quite simple (hence overly stringent) hypotheses is because we are just interested to show that the main idea discussed in this paper does indeed work.

\section{Outlook}

Clearly the kind of approaches illustrated above via the detailed treatment of two specific examples can be applied much more widely: it will be particularly interesting to do so in specific applicative contexts. 
A natural point of departure for such applications are isochronous systems, namely models whose generic solutions - in their entire natural phase space, or in open, hence fully dimensional, regions of it - are completely periodic (i. e., periodic in all their degrees of freedom) with the same fixed period (independent of the initial data, provided they stay within the isochrony region). As recently pointed out (see for instance [18]), quite a lot of dynamical systems can be modified so that they become isochronous, entailing the conclusion that isochronous systems are not rare. Each of these isochronous systems can then be further extended - along the lines obviously suggested by the treatment detailed above, see in particular the specific case treated in Section 3 - in order to generate classes of asymptotically isochronous systems, namely systems featuring open, hence fully dimensional, regions in their natural phase space (possibly including all of it) in which all (or almost all) their solutions display asymptotically a completely periodic behavior with the same fixed period, see (1). The technique to manufacture such generalized systems is clearly suggested by the examples treated above: of course these systems could be autonomous, as the examples treated above, or they might feature an explicit time-dependence, as could have been included in the system treated in Section 3 by assuming the functions $F$ and $f$ to also feature an explicit time dependence (but autonomous systems are generally more interesting than nonautonomous ones).

Often the natural context to investigate isochronous systems is in the complex rather than the real $[18,20]$ - although every system with complex dependent variables can of course be reformulated as a system with twice as many real dependent variables. Hence it may be of interest to mention how the findings detailed in Section 3 would be affected if the dependent variables $x_{n}$ and $w$ in the model (14) were allowed to be complex keeping of course real the time $t$ and positive the constant $\omega$, while the constant $g^{2}$ could now also be complex. It is then well known $[18,20]$ that the isochronous character of the motions still prevails for the (integrable indeed solvable) many-body problem (14a) without the $F$ term (i. e., with an identically vanishing $F$; see (19)) - describing motions taking place in the complex $z$-plane rather than on the real line. But in the complex context the isochronous behavior is a bit different than in the real context: the phase space is then divided into sectors separated by lower-dimensional manifolds characterized by solutions which hit a singularity at a finite time due to a particle collision; an event forbidden in the real case with positive $g^{2}$, when the particles move on the real axis and the two-body force, singular at zero separation, is repulsive, see (14a), but which can happen in the complex case, although not for generic initial data. In the different sectors the motion is still completely periodic, but with different periods, characterizing each sector and being (generally rather small [22]) integer multiples of the basic period $T$, see (4a). Accordingly, the generic solution of the (generally nonintegrable) generalized model (14) will be nonsingular throughout its time evolution and it shall eventually settle within a sector, approaching asymptotically one of the completely periodic solutions in that sector of the (integrable) model (14a) with identically vanishing $F$.

A somewhat analogous outcome obtains for the model analogous to (14) but with (14a) replaced by

$$
\ddot{z}_{n}+\frac{1}{4} \omega^{2} z_{n}=\sum_{m=1, m \neq n}^{N}\left[g_{n m}^{2}\left(z_{n}-z_{m}\right)^{-3}\right]+F(w, \underline{z}, \underline{\dot{z}}), \quad n=1, \ldots, N
$$


featuring $N(N-1)$ different coupling constants $g_{n m}^{2}$ acting among every particle pair. In this case the model without $F$ is generally not integrable, yet (if considered in the complex, namely without restricting the dependent variables $z_{n}$-nor, for that matter, the coupling constants $g_{n m}^{2}$ - to be real) it still does feature an open, hence fully dimensional, region in its phase space where all solutions are completely periodic with the same period $T$, see (4a) $[18,24]$; while in other regions of its phase space it might also be periodic but with periods $\tilde{T}=p T$ where the numbers $p$ are integers but might be very large, or it might even display an aperiodic, quite complicated (in some sense chaotic) behavior [25] (for recent progress in the understanding of this phenomenology see [26-29]). It then stands to reason that the solutions of the generalized model (14) with (14a) replaced by (25) (and of course $\underline{x}$ in $(14 \mathrm{~b})$ replaced by $\underline{z}$ ) shall again approach asymptotically solutions including, from open regions of initial data, completely periodic ones - of the model (25) without F: entailing a remarkable, and quite rich, phenomenology. Clearly our motivation to mention this specific model is because of its prototypical role: indeed, the main aspects of this phenomenology shall also characterize the large class of isochronous (but by no means necessarily integrable) systems that can now be manufactured [18], once they are extended by adding to their equations of motion other, fairly general, terms having the property to disappear asymptotically (as $t \rightarrow+\infty$ ), as a consequence of the very dynamics implied by these extended equations of motion.

In conclusion let us re-emphasize that these results (as indeed all mathematically correct findings) might well be deemed remarkable or trivial, depending on the level of understanding of the reader. Once their foundation is understood, it becomes obvious how they can be extended to many other models - suggesting an ample applicative potential. But these developments exceed the scope of this paper.

\section{Acknowledgements}

One of us (FC) would like to thank François Leyvraz for several illuminating discussions. The research reported in this paper has profited from visits by each of the two authors in the Department of the other performed in the framework of the exchange program among our two Universities. The research of DGU is supported in part by the Ramón y Cajal program of the Ministerio de Ciencia y Tecnología and by the DGI under grants FIS2005-00752 and MTM2006-00478.

\section{References}

[1] R. de la Llave, A tutorial on KAM theory, in: Smooth Ergodic Theory and Its Applications Seattle, WA, 1999, Proc. Sympos. Pure Math. vol. 69, (Providence:Amer. Math. Soc.) 2001, pp. 175-292.

[2] G. Gallavotti, Arnol'd's diffusion in isochronous systems, Math. Phys. Anal. Geom. 1 no. 4 (1999) 295-312.

[3] J. Chavarriga, M. Sabatini, A survey of isochronous centers, Qual. Theory Dyn. Syst. 1 (1999) 1-70. 
[4] J.-P. Francoise, Isochronous systems and perturbation theory, J. Nonlinear Math. Phys. 12 suppl. 1 (2005) 315-326.

[5] A.R. Chouikha, Isochronous centers of Lienard type equations and applications. $J$. Math. Anal. Appl. 331 no. 1 (2007) 358-376.

[6] J. Giné, J. Llibre, A family of isochronous foci with Darboux first integral, Pacific J. Math. 218 no. 2 (2005) 343-355.

[7] N.N. Nekhoroshev, The Poincaré-Lyapunov-Liouville-Arnol'd theorem, Funct. Anal. Appl. 28 no. 2 (1994) 128-129.

[8] C. Gonera, Isochronic potentials and new family of superintegrable systems. J. Phys. A: Math. Theor. 37 no. 13 (2004) 4085-4095.

[9] O. Chalykh, A.P. Veselov, A remark on rational isochronous potentials, J. Nonlinear Math. Phys. 12 (2005) 179-183.

[10] M. A. Rodriguez, P. Winternitz, Quantum superintegrability and exact solvability in $n$ dimensions J. Math. Phys. 43 (2002) 1309-22.

[11] S. Gravel, P. Winternitz, Superintegrability with third-order integrals in quantum and classical mechanics, J. Math. Phys. 43 (2002) 5902-5912.

[12] F. Calogero, F. Leyvraz, On a class of Hamiltonians with (classical) isochronous motions and (quantal) equi-spaced spectra. J. Phys. A: Math. Theor. 39 (2006) 1180324.

[13] M.C. Nucci, P.G.L. Leach, K. Andriopoulos, Lie symmetries, quantisation and cisochronous nonlinear oscillators, J. Math. Anal. Appl. 319 (2006), 357-368.

[14] F. Calogero, S. Graffi, On the quantisation of a nonlinear Hamiltonian oscillator, Phys. Lett. A 313 (2003) 356-362.

[15] J. Dorignac, On the quantum spectrum of isochronous potentials. J. Phys. A: Math. Theor. 38 no. 27 (2005) 6183-6210.

[16] F. Calogero, F. Leyvraz, General technique to produce isochronous Hamiltonians, $J$. Phys. A.: Math. Theor. 40 (2007) 12931-12944

[17] F. Calogero, Isochronous systems, in: Encyclopedia of Mathematical Physics edited by J.-P. Françoise, G. Naber and Tsou Sheung Tsun (Oxford: Elsevier) 2006 (ISBN 978-0-1251-2666-3), vol. 3, pp. 166-172.

[18] F. Calogero Isochronous systems (Oxford: Oxford University Press) 2008.

[19] F. Calogero, D. Gómez-Ullate, A new class of solvable many-body problems with constraints associated with an exceptional polynomial subspace of codimension two, J. Phys. A: Math. Theor. 40 (2007) F573-F580.

[20] F. Calogero, Classical Many-Body Problems Amenable to Exact Treatments (Lecture Notes in Physics Monograph Vol. 66) 2001 (Berlin: Springer) 
[21] F. Calogero, Motion of Poles and Zeros of Special Solutions of Nonlinear and Linear Partial Differential Equations and Related "Solvable" Many Body Problems, Nuovo Cimento 43B (1978) 177-241.

[22] D. Gómez-Ullate, M. Sommacal, Periods of the goldfish many-body problem J. Nonlinear Math. Phys. 12 Suppl.1) (2005) 351-62.

[23] E. Hairer, S. P. Norsett, G. Wanner, Solving Ordinary Differential Equations I: Nonstiff Problems 1987 (Berlin: Springer) pp. 193-195

[24] F. Calogero Periodic solutions of a system of complex ODEs, Phys. Lett. A293 (2002) $146-150$.

[25] F. Calogero, M. Sommacal, Periodic solutions of a system of complex ODEs. II. Higher periods J. Nonlinear Math Phys. 9 (2002) 483-516.

[26] F. Calogero, D. Gómez-Ullate, P. M. Santini, M. Sommacal, The transition from regular to irregular motions, explained as travel on Riemann surfaces J. Phys. A: Math. Gen. 38 (2005) 8873-8896.

[27] F. Calogero, D. Gómez-Ullate, P. M. Santini, M. Sommacal, Towards a theory of chaos explained as travel on Riemann surfaces, J. Phys. A: Math. Gen., in press.

[28] Yu. Fedorov, D. Gómez-Ullate, Dynamical systems on infinitely sheeted Riemann surfaces, Physica D 227 (2007) 120-134.

[29] P. Grinevich, P. M.Santini, Newtonian dynamics in the plane corresponding to straight and cyclic motions on the hyperelliptic curve $\mu^{2}=v^{n}-1, n \in \mathbb{Z}$ : ergodicity, isochrony, periodicity and fractals, Physica D 232, (2007) 22-32. 